\title{
Smart Meter using Big Data in IoT
}

\author{
Somalina Chowdhury, Santanu Kumar Sen
}

\begin{abstract}
Nowadays Green energy or energy efficiency has become one of the key concerns of the people. In this era Smart Grid with Internet of Things has took a vital role. Here distributed system with Smart Grid principle is being discussed. Unlike traditional Grid, Smart Grid are bidirectional in nature. One of the important component of Smart Grid is Smart Meter. In this paper we will focus on the vast data handled by Smart Meter using Big Data. The paper will focus on efficient energy management and how tactical decision making is done by Big Data to improve the overall Smart Grid performance. Data is collected through sensors especially wireless sensors are used. A vast amount of data is collected, analyzed, and processed to retrieve information. This will increase the business prospects and will be cost effective in future. Issues like instability, blackouts, etc will be under controlled. In traditional process of meter reading collecting usage and generating bill is the vital issue done by manually visiting the individual location which is now automated. Smart Meter works with real time data. It will be shown how Big Data will improve customer relation as well as improves social welfare. Thus proper techniques of data mining is used to retrieve data but with high data security. Mesmerising of various current technology is done here to get ultimate information about energy consumption and also to maintain a balance among customers and utilities.
\end{abstract}

KEYWORDS: Smart Grid, Smart Meter, Internet of Things (IoT), Big Data, Green Energy, Energy consumption, Wireless Sensor Network (WSN), Wireless Sensor, Business Intelligence

\section{INTRODUCTION}

In traditional system every house in each apartment are having meter which can be digital or analogue, connected with individual appliances. An electricity board official, physically visit individual houses to check the meter reading each month and generates a manual bill for each month. But Smart meter smarten the entire system. IoT is one of the new technologies that make a Smart Meter as well as Smart grid smarter. IoT is a connection between internet and actual things. Here IoT connected to Smart grid infrastructure thus collecting a huge volume of data from simple home appliances of individual houses in a locality or city or may be across the border. As large data needed to handle, so Big data is introduced [1]. Big data has seven v's namely Volume, Velocity, Variety, Variability, Veracity, Visualizati on, and Value. As a large real time data is collected each time from customer using Smart meter, are need to process efficiently. Unless proper processing done, exact informations cannot be extracted from them. The demands of electricity supply energy balanced using Smart grid and renewable energy.

Revised Manuscript Received on October 20, 2020.

* Correspondence Author

Somalina Chowdhury*, Assistant Professor Principal Dept. of Computer Applications, GNIT Kolkata, West Bengal

Prof. (Dr.) Santanu Kumar Sen, Assistant Professor Principal Dept. of Computer Applications, GNIT Kolkata, West Bengal

(c) The Authors. Published by Blue Eyes Intelligence Engineering and Sciences Publication (BEIESP). This is an open access article under the CC BY-NC-ND license (http://creativecommons.org/licenses/by-nc-nd/4.0/)
Renewable energy are like energy produced from wind, solar, biomass, water can also called green energy, meaning they're naturally replenished. A massive positive change is thus observed. A large centralised electricity generator is distributed to various small generators which are selfindependent. These small generators can be made of renewables energies. Here the concept of distributed system is actually refer to the various utilities and power generation stations used to distribute power. This is a complex process done Big Data. And distributed generations refers to different power manufacturing source. The motive behind is to create enough power storage in the grid to make it more efficient. In traditional Grid electricity flow in one track from utility to customer. But in smart Grid energy flows in two ways or bidirectional track. Generally data are collected from Smart Meter with the help of sensors which are in general nowadays wireless in nature. Only collection of data is not enough, also data analysis is a vital part which is done by Big Data. Proper classification of Big Data is discussed here. Data analytics normally finds some meaningful informations from raw data. Big Data helps in structuring the vast amount of raw data in the system. Thus most of the utility inclined to the big data analytics as it improving their business and also provides security. Customers also shown interested in this new Smarter module of Smart meter with Big Data and IoT support, as their bill get reduced and can track their consumption at regular intervals in a secured manner. It has been seen instability and blackout gets reduce by this process.

\section{RELATED WORK}

Today consumption of energy is one of the basic concern and it gets complimented with IoT and Big Data. This is being designed, implemented and tested using an embedded system with Arduino and it takes renewable energy as source. Day by day increase in energy demands also increases energy cost and energy management becomes very important. A smart meter can serve this purposes with the help of Internet of Things (IoT) and Big Data in the commercial, industrial and as well as residential sector. Business intelligence is also can be blended with Smart Meter and utility company applications [2]. Smart Grid has many Big Data applications in different fields like Smart building, electric vehicles, data hub of utilities, etc., among which we will discuss about Smart Meter in this paper. Micro grid is also used to increase productivity and electricity consumption for enhancement of the overall efficiency. Smart Grids are one of the most prominent future technologies. Its Advanced Metering Infrastructure (AMI) creates a hub for Smart Grid components, and contains smart meters and sensors, among

Published By:

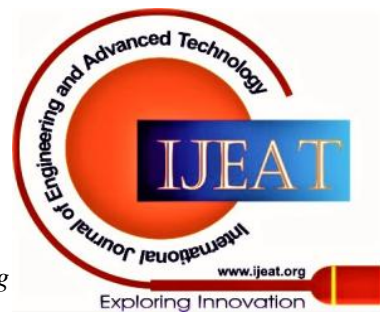

Blue Eyes Intelligence Engineering and Sciences Publication (C) Copyright: All rights reserved. 


\section{Smart Meter using Big Data in IoT}

which most of them are Wireless Sensors, to collect data. These data are collected and analysed through Big Data. Later these data are stored in cloud [3]. Big Data has its own vibrant features which can very efficiently and effectively handle large data volume [4]. Big data works with real time data and can be sense, process and represent data graphically with proper visibility. A demo environment was created and tested where electricity consumption of each house can be seen individually for each appliances in a building [5]. In this paper we will see how big data can be used in collecting data from smart meter, process and store the data. And also how we can analysis and get valuable results using this concept.

\section{METHODOLOGY}

The paper is capable of analysis and formatting the high volume of data collected in Smart meter. The smart grid collects a large amount of data from variant sources and stores it. Origin of the Big data in grids are utility data hub and secondary data centres [6]. Utility data hub includes PMU, SCADA, AMI, Smart meter etc. Secondary data centres which actually takes decision includes data derived from real life like GPS, sensor, database, digital documents etc. After collection data from various sources, in various forms acquisition is done, where parsing and validation of data is done to retrieve a quality information. Python and Java both can be used for this purpose. Storing data is the next step where data stored as per Big Data storage pattern. Data Processing and analytical stage is the heart of a big data system. Big data with no analysis has no value. Then data are analysed and processed, which require real time data processing. This can be done by IoT with the help of python. Finally the data stored used to take appropriate decision. The main importance of Big Data is to retrieve essential information from raw data and also reduce the complexity of data hiding.

Managing smart grids to generate power in smart way includes IoT to get accurate, consistent and reliable information from extremely large data pool for decision making shown in Fig 1. Thus makes the system reliable, efficient, fault tolerance and flexible. Analytics can be descriptive, diagnostic, predictive, and prescriptive models. These can be implemented in smart grid to satisfy customer by enhancing market based customer service.

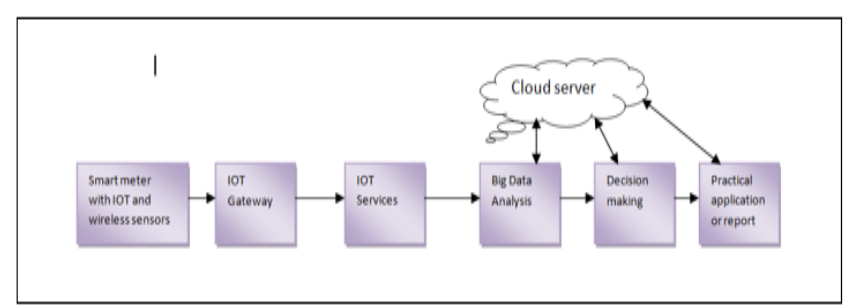

Fig 1. Relation of Smart meter with big data and cloud

\section{A. Smart Meter}

It is one of the key components of Smart Grid, which is use to track the electricity consumptions within a house. Manual collection of meter data reading, approx estimation of consumption on monthly basis and generating bill are not required as everything is automated. Consumption on daily basis and of each appliances can be monitored and thus bill can be reduced. It reduces the manual effort as well as give exact consumption by a user. Thus over billing is decreased. Customers and the utilities both are therefore very much positively affected by implementation of Smart Meter [7].

\section{B. Big data in smart grid}

Smart Grid deals with large amount of data having different types of structured or ordered, unstructured or standard and semi structured or unorganised forms that needs proper processing in effective and essential manner to derived tactical decisions [8]. A distributed flow has three levels: primary, secondary, and tertiary. Primary level concern with reliability in case of failure. Secondary modifies power quality while ternary replaces secondary to balance power and demands.[] It mines data with latest technologies to make high quality, reliable and interoperable smart grid operation. This can be achieved by big data. Big data depends on data mining, analysis and storage. Big data have $9 \mathrm{~V}$ features for five different functions in Smart grid [9]. Shown in Fig 2.

For collecting data:

- Veracity : It is the quality of the data and also data cleanup from pool of raw data collected at every time interval in proper efficient way

- Variety : Different types and structures of data collected by different sensors at one place

For processing data:

- Volume - Large amount of data after every interval generally after every $15 \mathrm{~min}$

- Velocity - Faster created and generated data as per response

For integrity data

- Validity : Authenticated, correct data to take proper decision

- Variability : With velocity consistency of the data get reduced during intervals

- Volatility : Data restore is one of the greatest factors

For visualization of data

- Visualization : Proper graphical representation of data

Use of data

- $\quad$ Value - Here actual information is being extracted from the data

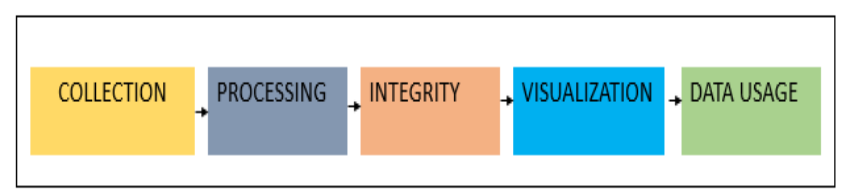

Fig 2. V's of Big Data

\section{Internet Of Things (IoT)}

IoT is a connection of various real life things to the internet. This technology have various domains like health monitoring, industrial systems, transport system, military systems, Electrical systems and many others. Day by day no of connected devices in Smart grid increases and thus a massive control is needed to connect them in internet and not only analysis but also managing them. For this we need IoT. And IoT needed high secure network in Smart grid infrastructure [10].

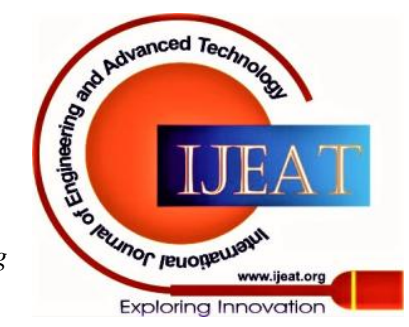


One of the complex process is to monitor, control and electric facility management which can be efficiently done by IoT. Smart grid contains large volume of data with different format and stored in different storage. As amount of data increases thus effectiveness of it also increases. Now these data collected through sensors, transmitted through proper secure connectivity. They are stored and processed in cloud with additional actions. All these work is done by IoT mechanism.

\section{Proposed Process}

Smart meter along with Big data can be used in Smart grid to improve its performance.

Here we will use IoT analysis of Big Data instead of cloud as we can then work with distributed data. Online dealing with the Big Data is the biggest challenge for electric energy sector. Response time of this analysis is high. Here given step by step processing of the entire system. Shown in Fig 3. IoT generates data, Big data processed data, cloud stores data.

- Data generated after every 15 min interval rate using IoT

- Data is collected from Smart Meter or wireless sensors in the grid

- Data transmitted through secured channel

- Automated consumption data reading send to utility at every interval

- Unstructured, structured, semi structured data analysed

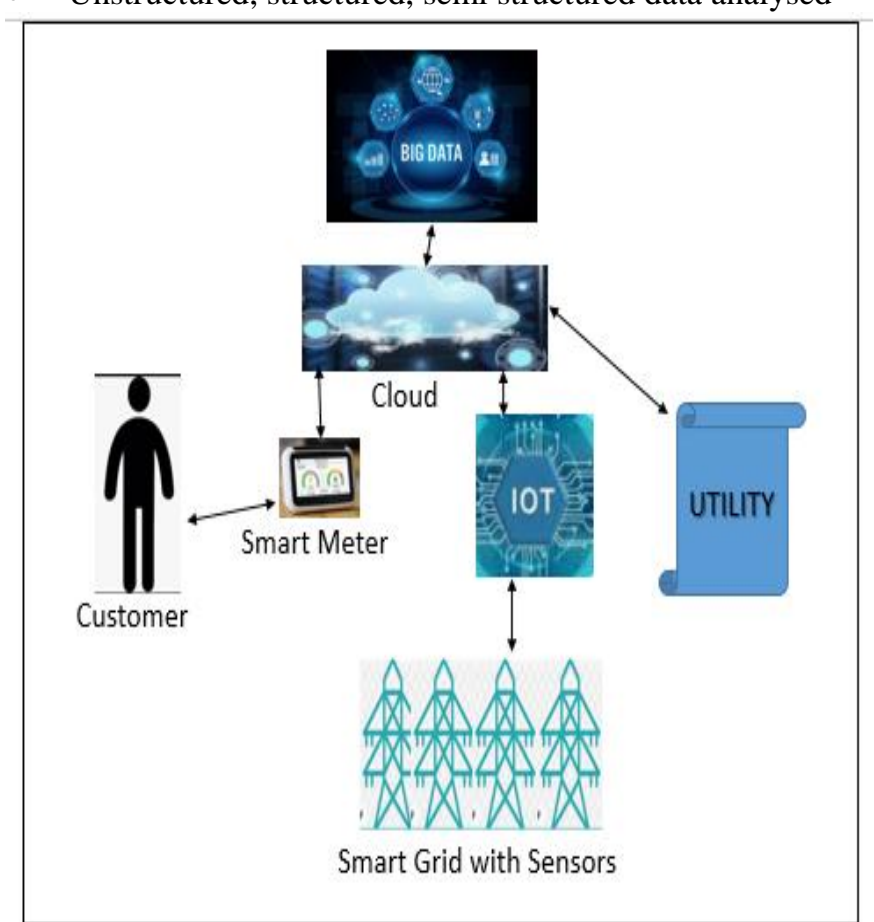

Fig 3. Process of Smart meter working with Big Data and Cloud

- Data is cleaned by dealing with missing or abnormal value

- Data integrated and transformed to information

- Data transformed to visualization format

- All these done by big data analysis process

- Data stored as big data format in cloud server

- Utility and customer both can download the data or report from their end
- That is individual appliances electricity consumption or day wise consumption report can be generated

- Thus enhances business model with customer satisfaction

\section{RESULT AND DISCUSSION}

In this paper we have analysis how big data have improved the performance of Smart Meters. Since IoT implemented Smart meter produced large amount of real time data. And as they can be of any form so big data smoothen its Performance.

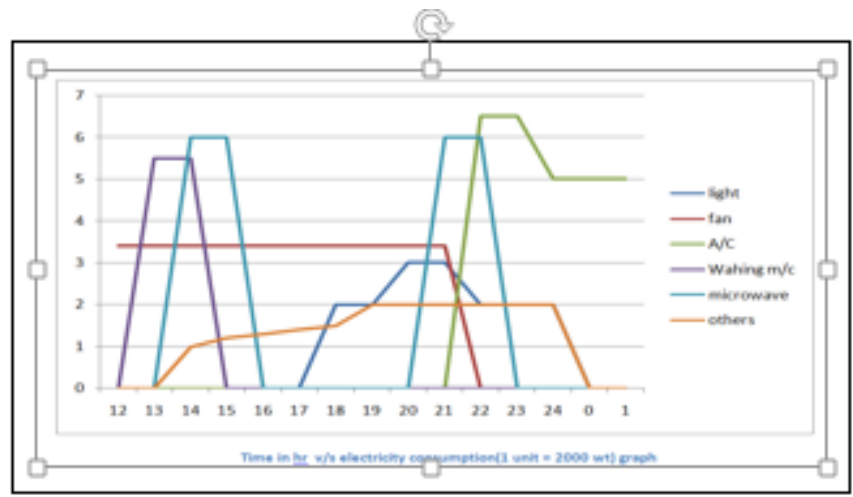

Big data can handle large amount of data with accuracy, proper efficiency and maintains its integrity. And due to proper management of data easily reports of electricity consumption reading and cost of each appliances of each house or building can be generated. . As Smart grid are bidirectional are in nature so energy wastage is reduced and also reports can be generated from both utility and customer ends.

\section{CONCLUSION}

In upcoming days Smart Meter will be one of the high priority research area. In this paper it is discussed that how wan secure the data in Smart Meter in Smart Grid through big data. One of the main challenges is to gather data, achieved by Big Data. BD create best tactical decisions. And a way to securely communicate and preserved the data. Another challenge is related to security in cyber space and privacy of the sensitive data. The proposed technique shown here is really a good approach to build a balanced communication with customers and utility companies. Thus we can use Smart meter with advancement.

\section{REFERANCES}

1. Yang Zhang, Tao Huang and Ettore Francesco Bompard," Big data analytics in smart grids: a review ", in Zhang et al. Energy Informatics (2018) https://doi.org/10.1186/s42162-018-0007-5

2. Prachi Kulkarni, D.K. Chitre," Energy Consumption Using IoT and Big Data Analytics Approach in Smart Home ". In IJIRSET

3. Driss Benhaddou, Mohamed Riduan Abid, Ouidad Achahbar , Nacer Khalil, Tajjeeddine Rachidi and Maen Al Assaf," BIG DATA PROCESSING FOR SMART GRIDS ", in IADIS International Journal on Computer Science and Information Systems Vol. 10, No. 1, pp. 32-46 ISSN: 1646-3692

Published By:

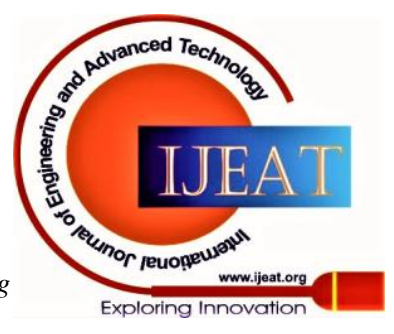

Blue Eyes Intelligence Engineering and Sciences Publication (C) Copyright: All rights reserved. 


\section{Smart Meter using Big Data in IoT}

4. Laura L. Pullum and IEEE Smart Grid Big Data Analytics, Machine Learning and 10 Artificial Intelligence in the Smart Grid Working Group, "Big Data Analytics in the Smart Grid"' by IEEE team of Smart grid

5. A.Berouine, F. Lachhab, Y. Nait. Malek, M. Bakhouya, R. Ouladsine, "A Smart Metering Platform Using Big Data and IoT technologies", by 2007 IEEE

6. P.C. Chen, T. Dokic, and M. Kezunovic, "The use of big data for outage management in distribution systems," in Int. Conf. Electricity Dist. (CIRED) Workshop, 2014

7. Shibily Joseph, and E. A. Jasmin,"Stream computing framework for outage detection in smart grid.", International Conference on Power, Instrumentation, Control and Computing, 2015.

8. Suhail Sami Owais, Nada Sael Hussein, "Extract Five Categories CPIVW from the 9V's Characteristics of the Big Data ", in International Journal of Advanced Computer Science and Applications, Vol. 7, No. 3, 2016

9. SomalinaChowdhury, Santanu Kumar Sen, "Security in Smart Meter using Iot", in IJEAT, ISSN: 2249 - 8958, Volume-9 Issue-4, April 2020

10. SomalinaChowdhury, Santanu Kumar Sen,, "Designing of Smart meter in Smart grid using IoT ",Processing in Manufacturing and Quality Engineering - Industrial Perspective

\section{AUTHORS PROFILE}

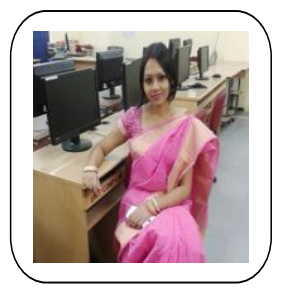

Somalina Chowdhury, More than 8 years of experience in the field of Computer Application. Pursuing $\mathrm{PhD}$ in Computer Science and Engg.(CSE)/Information Technology(IT) from MAKAUT, West Bengal since Dec 2018. Completed MCA and BCA from MAKAUT in 2008 \&2011 respectively. Member of Institution of Engineers (India).Received Believers Award from National Program on Technology Enhanced Learning (NPTEL) for completion of four courses in a single semester. One patent is filed. Presently working as Assistant Professor in Guru Nanak Institute of Technology (GNIT), Kolkata since 2011. Awarded for organizing Smart India Hackathon, 2019. Areas of working interest are IoT, Cloud Computing, Big Data and Genetic algorithm, Network Security, Cryptography and Wireless Sensor Network. Around 9 papers in international journal among them one in IJEAT.

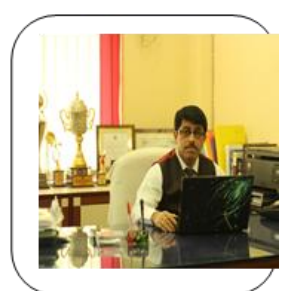

Prof. (Dr.) Santanu Kumar Sen, Around 25 years of experience (8 years in Industry and 17 years in Engineering Academia including Abroad). PhD (Engg.), MBA, M.Tech, B.E, Chartered Engineering. Professional Engineer, FIET (in process), UK Fellow: FIETE, FIE, SMIEEE, USA Senior Member- (SMCSI), India Life Member LMISTE, India Senior MemberMACM, USA. Awarded the 1st Professional Engineer Degree under Computer Science Division from IE in 2019. Expert Member of International Professional Engineering under CS from IE in 2019 Board Member of WBJEEB since 2015 Advisor, National Cyber Security Cell, New Delhi.Rashtriya Shiksha Gourav Puroskar from Centre. "Bharat Bibhushan Samman Puraskar" from EHRDA, New Delhi in 2013. Research Paper Publications: 70+ Patents filed and Published: 6 Work Experience: Presently working as Principal of Guru Nanak Institute of Technology Guru (An Autonomous Degree Engineering College) since 2012.

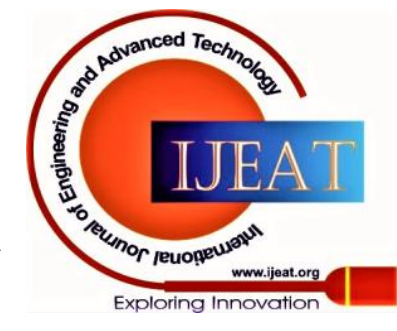

